\title{
Pars Plana Vitrectomy and Internal Limiting Membrane Peeling in Epimacular Membranes: Correlation of Function and Morphology across the Macula
}

\author{
Wolfgang J. Mayer Michaela Vogel Aljoscha Neubauer Marcus Kernt \\ Anselm Kampik Armin Wolf Christos Haritoglou \\ Department of Ophthalmology, Ludwig Maximilian University Munich, Munich, Germany
}

\section{Key Words}

Spectral domain optical coherence tomography · Epiretinal membrane - Macular pucker . Photoreceptor layer .

Microperimetry $\cdot$ Inner and outer segment

\begin{abstract}
Purpose: To analyze the correlation between morphological and functional results 12 months after epiretinal membrane (ERM) surgery. Methods: 31 eyes from 31 consecutive patients with metamorphopsia and best corrected visual acuity (BCVA) below 20/32 underwent a transconjunctival 23gauge vitrectomy with ERM and internal limiting membrane peeling. Preoperatively and 3, 6 and 12 months postoperatively, we assessed BCVA, microperimetry (MP-1) and spectral domain optical coherence tomography (SD-OCT). Photoreceptor inner and outer segment (IS/OS) was graded on SD-OCT images and correlated with microperimetry measurements in the fovea and parafoveal region. Results: The postoperative BCVA was significantly better in eyes with an intact IS/OS junction $(p<0.01)$. In addition, the mean defect depth was postoperatively decreased in the foveal and parafoveal area in eyes with an intact IS/OS junction. A correlation of SD-OCT IS/OS images and microperimetry in eyes with improvement in BCVA of at least 2 lines revealed a statistically significant result for the parafoveal quadrants $(p<$
\end{abstract}

0.011 for SD-OCT and $\mathrm{p}<0.005$ for microperimetry) but not for the foveal area alone. Conclusions: The IS/OS regeneration in the parafoveal quadrants contributes significantly to the recovery of BCVA following ERM surgery. Consequently, functional and morphological tests of the macular area should not be limited to the fovea but should be extended to the parafoveal region.

Copyright $\odot 2013$ S. Karger AG, Basel

\section{Introduction}

Epiretinal membrane (ERM) formation is characterized by a proliferation of glial cells and hyalocytes in the macular area [1]. In most cases, the disease is idiopathic, but can also be seen following retinal detachment surgery, cryotherapy or laser photocoagulation. In the elder population, the prevalence is about $70 \%$ [1]. As the epimacular membrane contains contractile cellular elements, the disease may be associated with retinal folding and macular thickening, leading to a decrease in visual acuity (VA) and disturbing metamorphopsia, photophobia and micropsia [2-4].

The current surgical approach includes a pars plana vitrectomy and removal of ERM. Some surgeons also remove the internal limiting membrane (ILM) as a prophy-

\section{KARGER}

E-Mail karger@karger.com

www.karger.com/oph
(C) 2013 S. Karger AG, Basel

0030-3755/13/2301-0009\$38.00/0 
lactic measure in order to prevent cellular reproliferations. Vital dyes such as trypan blue or brilliant blue can be used to visualize the epiretinal tissue and the ILM, respectively [5].

The current progress in retinal imaging using highresolution spectral domain optical coherence tomography (SD-OCT) enables us to capture detailed, pseudohistological images of all retinal layers. Having these highresolution images available, the outer retinal layers, especially the junction between the photoreceptor inner and outer segment (IS/OS juncture), is now understood to play an important role in functional recovery following macular surgery [6-9] as recent studies have suggested [7, $8,10]$. While in healthy eyes the IS/OS juncture can be identified as a continuous hyperreflective band just above the retinal pigment epithelium, disruptions of the IS/OS juncture have been discussed as potential predictors for poor visual recovery in eyes having undergone macular surgery $[6,7,11-17]$.

It is known that tractional forces first lead to morphological disorganization of the inner retina [18-20] followed by changes in the outer retinal layers as the disease progresses. Many OCT studies in patients with ERM indicated prognostic factors, besides VA and duration of symptoms [21-23], such as marked loss of the foveal contour, the presence of cystoid macular edema, and the integration of the photoreceptor layer $[4,6,10,24,25]$.

In addition to these improvements and developments in retinal imaging, more detailed macular functional analyses are obtainable using microperimetry systems providing information on foveal fixation, macular sensitivity and depth of central macular defects. Funduscontrolled microperimetry is a functional test allowing for the analysis of retinal sensitivity with a high spatial resolution [26]. It differs from standard perimetry techniques as it is independent of eye movements during the examination. This is achieved by controlled projection of the test stimuli in constant topographic relation to retinal landmarks. An exact overlay of such functional mapping with high-resolution images of the retina may reveal the functional impact of microstructural alterations in vivo.

Previous reports $[7,14-17,27,28]$ in the literature dealing with a correlation of function and morphology using microperimetry and SD-OCT demonstrated that preoperative outer retinal disruption was associated with poorer visual recovery postoperatively. However, they did not differentiate between localized alterations seen in the fovea and potential abnormalities in the parafoveal region.

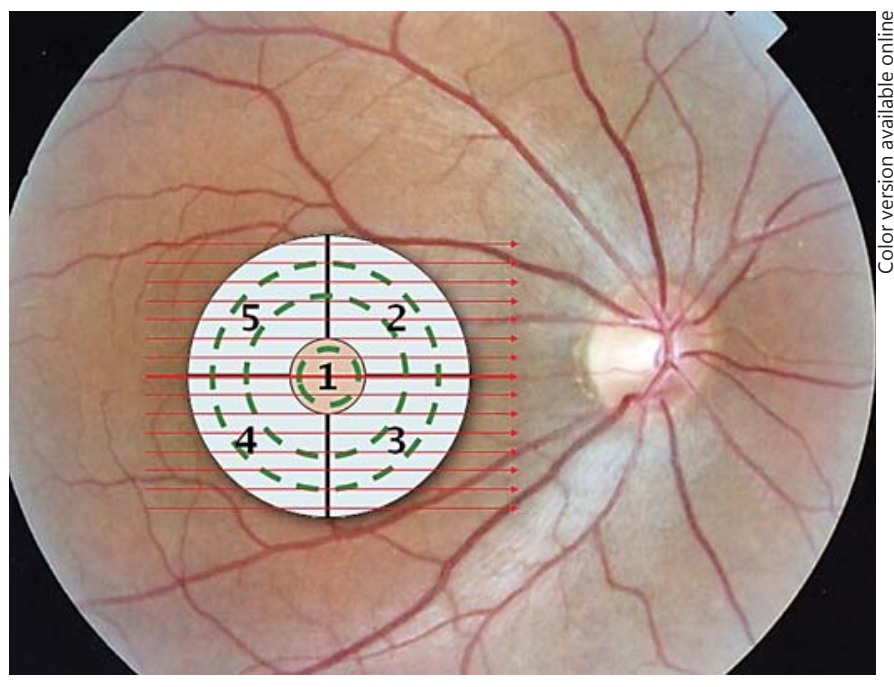

Fig. 1. Scheme of the individually analyzed 5 sectors (modified ETDRS grid): OCT scans (red), microperimetry (green).

Therefore, the aim of the present investigation was to analyze the correlation between photoreceptor IS/OS integrity as documented on SD-OCT and microperimetry findings both in the foveal and parafoveal area in eyes undergoing vitrectomy for epimacular membrane repair and to correlate findings with postoperative VA.

\section{Materials and Methods}

\section{Study Population and Surgery}

In this prospective, consecutive, observational nonrandomized study, 31 eyes of 31 patients with ERM formation were enrolled between July 2011 and January 2012. Informed consent was obtained prior to surgery. The study was approved by the Institutional Review Board. All patients suffered from metamorphopsia and decrease in best corrected visual acuity (BCVA). Surgery was performed in eyes with BCVA below 20/32, and/or persisting symptoms such as disturbing metamorphopsia or micropsia for a period of at least 6 months. Standard 23-gauge vitrectomy with peeling of the unstained ERM followed by the ILM was performed by two highly trained vitreoretinal surgeons (A.K. and C.H.). The ILM was visualized using an approved heavy brilliant blue solution (Fluoron GmbH, Neu Ulm, Germany). In patients with relevant lens opacification, a combined surgery of cataract surgery, intraocular lens implantation and pars plana vitrectomy was performed. Patients with prior vitrectomy, any history of uveitis or retinal detachment, recurrent ERM, proliferative diabetic retinopathy or patients with concurrent macular diseases such as macular degeneration or diabetic macular edema were excluded.

\section{Patient Examination}

Preoperatively and 3, 6 and 12 months after surgery, we performed the following examinations: measurement of BCVA, slit lamp examination of the anterior segment with documentation of 


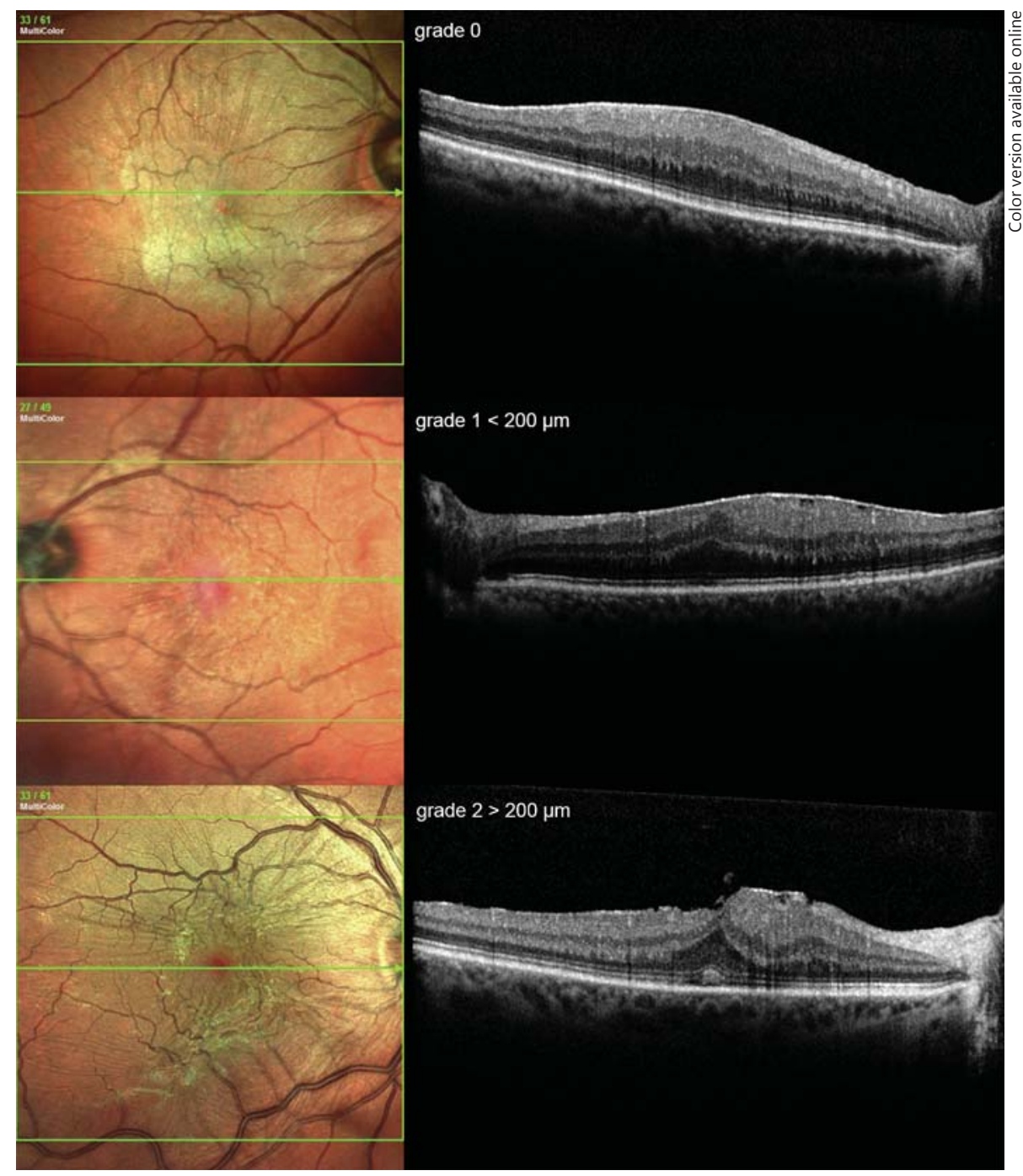

Fig. 2. Classification of SD-OCT photoreceptor IS/OS grading: grade 0 (intact IS/OS integrity), grade 1 (irregular IS/OS integrity $<200 \mu \mathrm{m}$ ), grade 2 (loss of IS/OS integrity $>200 \mu \mathrm{m})$.

lens opacification using the Lens Opacities Classification System III, funduscopy using a 78-dpt lens, central 10-degree microperimetry (Nidek MP-1, Nidek Instruments Inc., Japan) and SD-OCT (Heidelberg Engineering, Heidelberg, Germany). Measurements of the depth of the mean defect, mean sensitivity and VA results expressed as a gain in lines were correlated to morphological changes in the photoreceptor layer (IS/OS) as analyzed by SD-OCT.

In order to allow for a reliable and standardized correlation, the macular area was divided into 5 sectors according to the ETDRS protocol using the MP-1 software (modified ETDRS grid): sector 1 was defined as the foveal area while the parafoveal area was divided into 4 quadrants labeled sectors $2-5$ (fig. 1 ).

Microperimetry was performed using 4-2-1 staircase strategy with a Goldmann III stimulus and 40 stimulus locations covering the 10-degree center field. The differential luminance, defined as the difference between stimulus luminance and background luminance, was $127 \mathrm{~cd} / \mathrm{m}^{2}$ at $0 \mathrm{~dB}$ stimulation and the maximum stimulus attenuation was $20 \mathrm{~dB}$. The duration of the stimulus was 200 $\mathrm{ms}$ and the fixation target was varied in size according to the pa- tient's VA. There were 8 stimuli presented in the central 2-degree field, 24 stimuli covering the central 6-degree field; all in all, 40 stimuli were applied to cover the entire central 10-degree field. After conclusion of the fundus microperimetry, a colored fundus image was obtained and overlayed with the microperimetry results in the modified ETDRS grid.

For the SD-OCT analyses, a modified volume scan was performed in each observational time step. Five horizontal scans of the fovea and 7 horizontal scans of each parafoveal quadrant with a single scan distance of $11 \mu \mathrm{m}$ were obtained and evaluated in the modified EDTRS grid (5 sectors; fig. 1). The photoreceptor layer was imaged as a hyperreflective line showing the photoreceptor IS/ OS junction above the retinal pigment epithelium. The integrity of the IS/OS layer was evaluated by two independent blinded investigators (authors 1 and 6) and graded in all sectors using a software-defined grid layer and a grading score from 0 to 2 according to other studies $[7,17]$ : grade 0 was defined as an intact IS/OS junction as seen by a continuous hyperreflective line, grade 1 as a focal disruption of the IS/OS junction of $<200 \mu \mathrm{m}$, and grade 2 as a dis- 
Fig. 3. Correlation of functional and morphological findings using the example of a macular pucker (a) with recovery of the IS/ OS zone in the postoperative OCT (b). BCVA improved from $0.4 \log$ MAR before to $0.1 \log$ MAR after surgery.

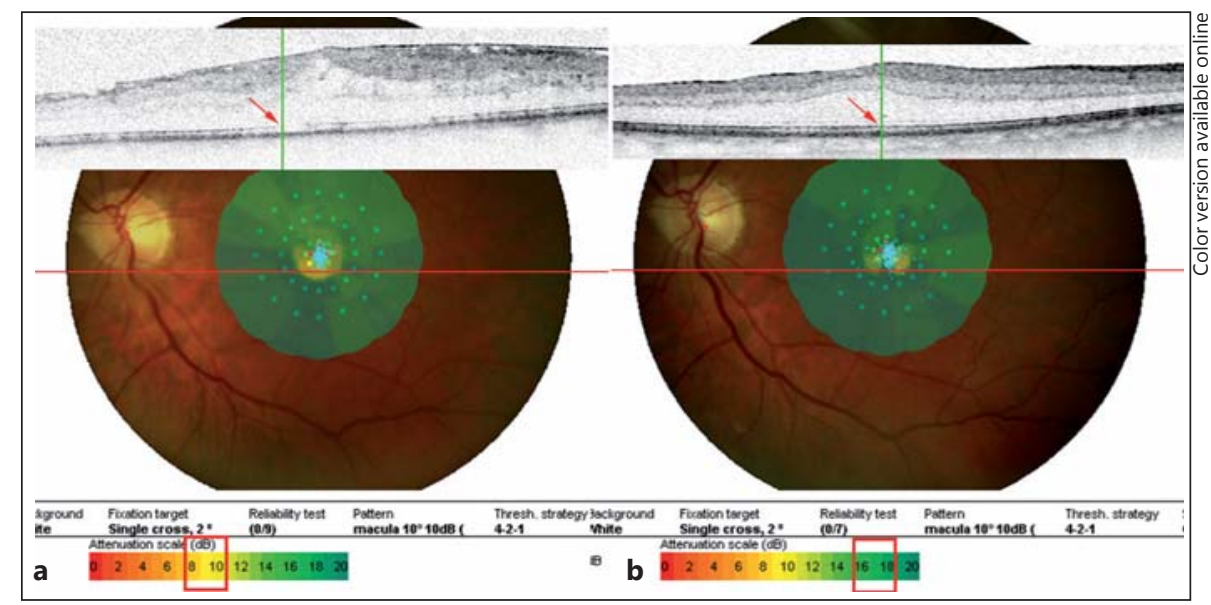

ruption of the IS/OS junction of $>200 \mu \mathrm{m}$ (fig. 2). OCT and microperimetry images were matched using the adapted integrated MP-1 software importing function and correlations between SDOCT IS/OS grading and MP-1 results were recorded in all 5 sectors using the modified ETDRS grid.

\section{Statistics}

All VA measurements (Snellen chart) were converted to the logarithm of the minimum angle of resolution (logMAR) VA. The Mann-Whitney test and cross-tables using Fisher's exact test were performed to compare logMAR values (dependent variable) to SD-OCT and microperimetry results (independent variables). A change of BCVA of at least 2 lines was considered statistically significant. The mean sensitivity of all 5 macular sectors (fovea and parafoveal area) was correlated to mean SD-OCT gradings (0-2). All analyses were conducted using the SPSS Statistics 19.0 Software (SPSS, Inc., Chicago, Ill., USA). A p value $<0.05$ was considered statistically significant.

The impact of lens opacities on BCVA was analyzed as follows: the values for pseudophakic eyes and clear lens were set as 0 in the Lens Opacities Classification System III. The significance of the differences between preoperative and postoperative BCVA was evaluated using the Mann-Whitney $U$ test. The possible correlation between the preoperative VA and the functional parameters was investigated using Spearman's rank correlation test.

\section{Results}

The study population consisted of 18 males and 13 females with a mean age of 68.6 years (range of 56-79 years).

The ERMs were successfully removed in all eyes. Combined cataract surgery and vitrectomy was performed in 9 eyes, 5 eyes were already pseudophakic. There were no postoperative complications in any of the cases, i.e. endophthalmitis, retinal detachment, macular edema or new ERM formation.
Table 1. Correlation between preoperative IS/OS grading and postoperative mean defect measurements in microperimetry

\begin{tabular}{|c|c|c|c|c|c|}
\hline \multirow{2}{*}{$\begin{array}{l}\text { Preoperative } \\
\text { IS/OS grading }\end{array}$} & \multicolumn{5}{|c|}{ Postoperative mean defect (12 months) } \\
\hline & sector 1 & sector 2 & sector 3 & sector 4 & sector 5 \\
\hline $\begin{array}{l}\text { Correlation }^{1} \\
\text { p value }^{2}\end{array}$ & $\begin{array}{r}-0.305 \\
0.095\end{array}$ & $\begin{array}{r}-0.523 \\
0.003\end{array}$ & $\begin{array}{r}-0.377 \\
0.040\end{array}$ & $\begin{array}{r}-0.517 \\
0.003\end{array}$ & $\begin{array}{r}-0.628 \\
0.000\end{array}$ \\
\hline
\end{tabular}

${ }^{1}$ Spearman's rank test.

${ }^{2} \mathrm{p}<0.05$ compared with baseline.

Overall, the postoperative improvement in BCVA was significantly more pronounced in eyes with a postoperative intact (grade 0) IS/OS junction on the SD-OCT images compared to those eyes with an irregular (grade 1) or disrupted (grade 2) IS/OS junction ( $\mathrm{p}<0.01$; fig. 3 ). In these patients, a significant increase in mean sensitivity and a significant decrease in the mean defect depth in microperimetry were noted postoperatively. No sign of intraretinal fluid or vitreoretinal traction was recorded during the whole observation period in all SD-OCT images.

\section{Correlation of Visual Improvement to SD-OCT and Microperimetry}

Overall, mean macular sensitivity increased from 14.6 \pm 2.3 to $16.1 \pm 2.1 \mathrm{~dB}$ in the foveal area and from $8.0 \pm 3.0$ to $14.3 \pm 4.3 \mathrm{~dB}$ in the surrounding 4 quadrants after 12 months. Comparing foveal (sector 1) and parafoveal (sectors 2-5) measurements, a significant correlation could only be found for the parafoveal area between mean defect and IS/OS junction before and after surgery (table 1;
Mayer/Vogel/Neubauer/Kernt/Kampik/ Wolf/Haritoglou 

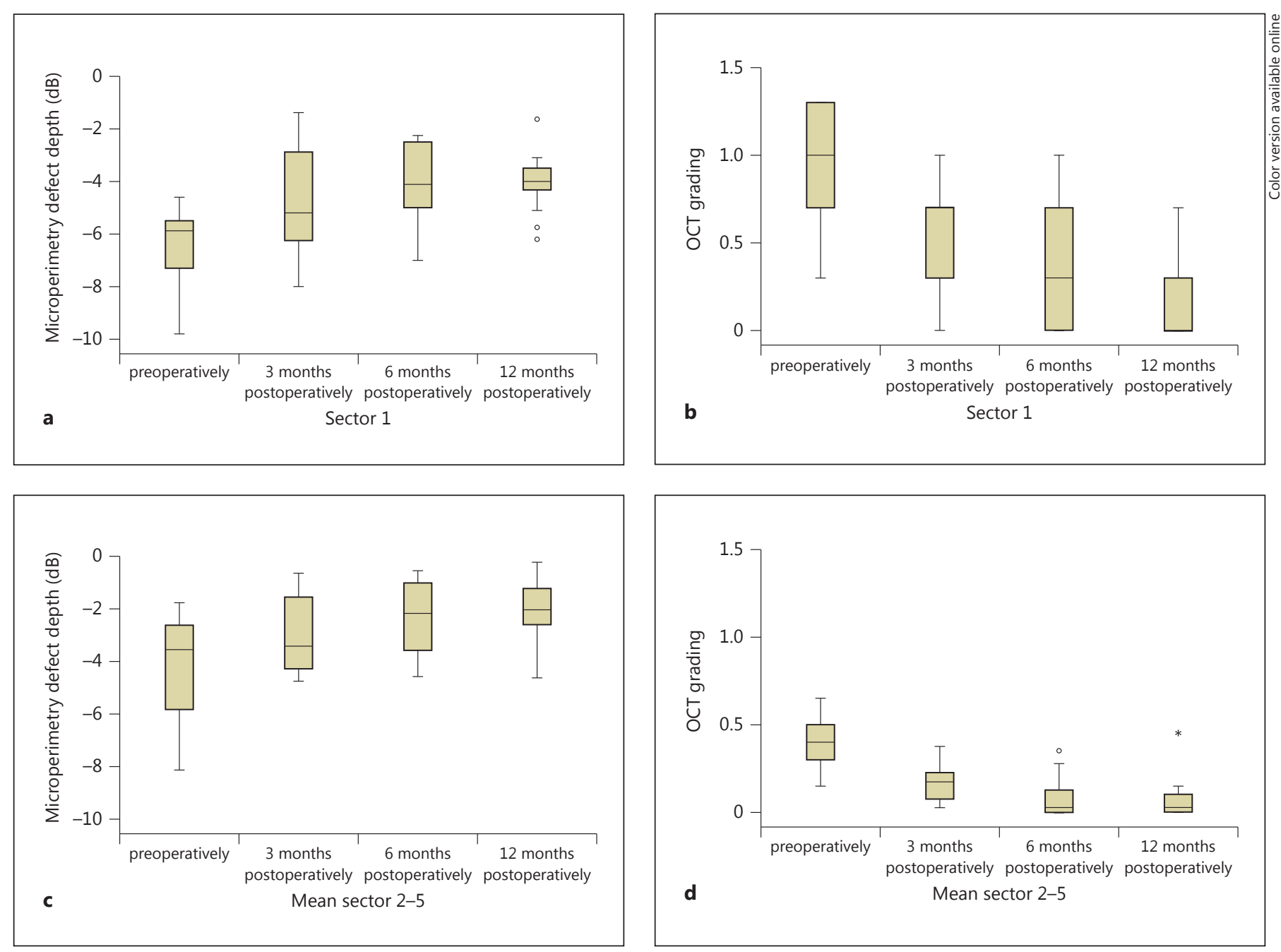

Fig. 4. a-d OCT grading and microperimetry (mean defect depth) results in the foveal (a, b; sector 1) and parafoveal (c, d; sectors 2-5) area preoperatively and after 3,6 and 12 months postoperatively. Note the decrease in the mean defect depth and the improvement in the IS/OS integrity.

fig. $4 \mathrm{a}-\mathrm{d}$ ). Table 1 demonstrates the results of Spearman's rank correlation test that revealed a significant correlation in the parafoveal sectors between the preoperative IS/OS integrity and the postoperative functional results in microperimetry analyses $(\mathrm{p}<0.05)$.

Patients with a postoperative VA improvement of 2 lines or more after 12 months $(n=20)$ showed a better preoperative IS/OS integrity (grade 0 ) in comparison to patients with a lower improvement in VA $(\mathrm{n}=11$; fig. 5). There were no significant differences in baseline data concerning VA, SD-OCT measurements and microperimetry analyses between the groups ( $\mathrm{p}$ for all parameters $>0.10$ ). Comparing patients with a significant improvement in BCVA of at least 2 lines at 12 months (group 1) after surgery to those with no significant improvement (group 2), no statistically significant difference concerning the regeneration of the IS/OS junction was noted when looking at the foveal scan alone $(\mathrm{p}=0.427$; fig. $6 \mathrm{a})$. In contrast, when assessing the parafoveal sectors $2-5$, a statistically significant correlation of visual gain and IS/ OS regeneration was observed, with a better IS/OS juncture regeneration in patients with 2 or more lines of BCVA improvement ( $\mathrm{p}=0.011$; fig. $6 \mathrm{~b}$ ).

Similarly, correlating the decrease in the mean defect depth in microperimetry to the development of VA, a statistically significant difference for both the foveal and parafoveal sectors was seen. The decrease in the mean defect depth was more pronounced in patients of group 1 
Fig. 5. Preoperative photoreceptor status compared to VA improvement after 12 months in the foveal (sector 1) and in the parafoveal (sector 2-5) quadrants. * = Mean number of patients in all 4 parafovealquadrants (sectors 2-5).

Fig. 6. Changes of the IS/OS grading after 12 months postoperatively comparing patients with BCVA improvement of 2 or more or less than 2 lines. No significant improvement is seen for a grading of the foveal scan alone (a). A significant improvement was seen when looking at the parafoveal sectors $2-5$ (b).

Fig. 7. Changes of the mean defect depth after 12 months postoperatively comparing patients with BCVA improvement of 2 or more or less than 2 lines. A significant improvement is seen for a grading of both the foveal scan alone (a) and the parafoveal scans (b). The result was more significant in the parafoveal area (sectors 2-5).
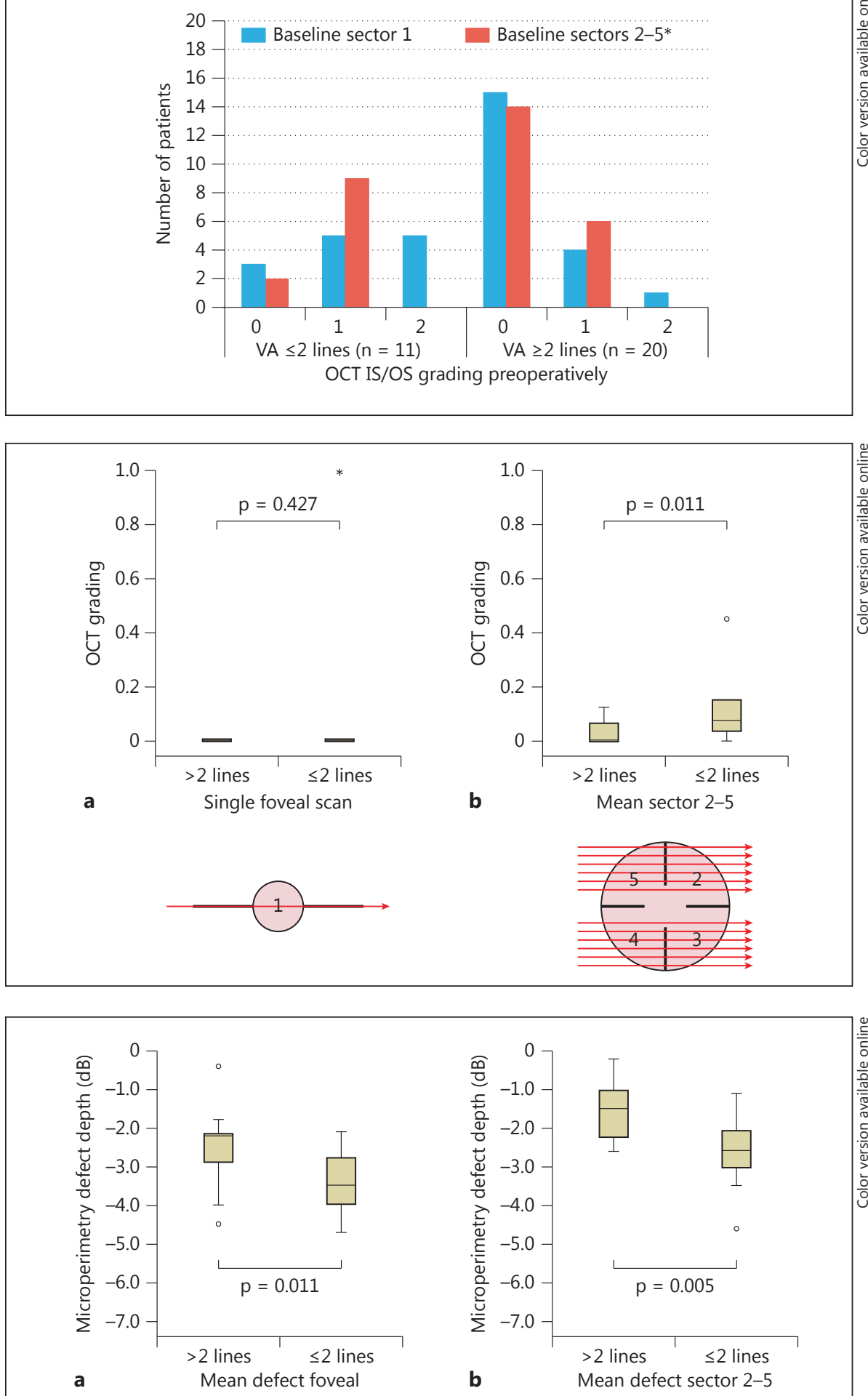
(VA gain of at least 2 lines). However, the result was more remarkable in the parafoveal sectors 2-5 compared to the foveal sector 1 ( $p=0.005$ vs. $p=0.011$; fig. $7 a$ and $b$ ).

No correlation could be established concerning retinal thickness and VA $(\mathrm{p}=0.375)$. The statistical analyses of mean values measured using the Lens Opacities Classification System III showed no significant influence of cataract formation on VA improvement postoperatively $(\mathrm{p}=$ 0.16 , data not shown). No patients underwent cataract surgery in the time of the follow-up period.

\section{Discussion}

ERM formation represents a pathology which is localized on the retinal surface or the vitreoretinal interface. Cellular proliferation is very likely related to an incomplete posterior vitreous detachment in idiopathic cases, but may also be the result of other conditions such as retinal breaks, laser or cryotherapy, inflammatory diseases or retinal detachment. Tractional forces first lead to morphological disorganization of the inner retina [18-20] followed by changes in the outer retinal layers as the disease progresses. It is very suggestive that the extent and localization of outer retinal changes in eyes with ERM formation may be very variable and correlate to the size of the membrane and the focus where tractional forces are most pronounced.

The described changes are often associated with a decrease in VA and disturbing metamorphopsia, which are the main indications for surgical intervention. As for other traction maculopathies such as macular holes or vitreomacular traction syndrome, surgery consists of a pars plana vitrectomy with peeling of visible epimacular membranes and the ILM $[2,29]$.

However, in some cases, the functional result obtained postoperatively is not satisfying both for the surgeon and the patient despite clinically visible anatomic success. The reason for this discrepancy is now understood to be correlated with alterations of the outer retinal layers, which can be depicted with high-resolution imaging technologies such as OCT, but not during a clinical examination using a 78- or 90-dpt lens during biomicroscopy [30].

Wakabayashi et al. [31] analyzed reconstructive changes following macular hole surgery and showed that the integrity of the back-reflection lines from the photoreceptor IS/OS junction and the external limiting membrane as seen on SD-OCT images may predict subsequent restoration of the foveal photoreceptor layer and the potential for better visual outcomes [31]. In addition to a positive

Vitrectomy in Epimacular Membranes correlation between outer segment restoration and functional results after macular surgery, Itoh et al. [12] observed that a recovery of the foveal cone microstructure may be seen as late as 12 months after anatomically successful surgery. These findings were supported by other authors $[2,4,7,10,16,17,25,28,29,32]$.

However, so far published studies investigating the role of the outer retinal layers and their impact on functional recovery focused mainly on foveal sections obtained during OCT examinations and did not include the area surrounding the fovea $[2,4,7,10,16,17,25,28,29$, 32].

In contrast to that, the present investigation systematically analyzed both the foveal and parafoveal region and established a correlation between morphological abnormalities detected in OCT images and retinal function in these specific areas as measured by microperimetry and BCVA. To the best of our knowledge, the present study is the first to demonstrate the relevance of the parafoveal area for postoperative VA improvement. Our results indicate that photoreceptor IS/OS integrity on SDOCT is a statistically significant predictor of VA in patients with ERM formation, and statistical analysis illustrates that IS/OS disruption increases the predictive power of OCT measurements. We conclude from our data that morphological and functional tests (SD-OCT and microperimetry) in patients with ERM formation should not be focused on the foveal region alone, but should also cover the parafoveal area. Therefore, with respect to the role of the parafoveal region in functional recovery as shown in the present investigation one may suggest that epiretinal tissue and potentially also the ILM should be removed as thoroughly and completely as possible in a large enough area.

From our point of view, a strength of this study is the combination of microperimetry and SD-OCT to assess the foveal and the parafoveal region, which allows for a more detailed evaluation of morphological abnormalities of the outer retina and their impact on retinal function. An analysis of foveal OCT scans and microperimetry measurements alone may not provide enough information on the effect of ERM formation both on the outer retinal layers and their functional relevance before surgery as well as their effect on postoperative functional development as measured by BCVA. We found a higher statistical significance at 12 months postoperatively following an analysis of the parafoveal region compared to the fovea using SD-OCT and microperimetry when correlating the results of these measurements to postoperative VA improvement. As a consequence, when using high- 
resolution imaging techniques and functional tests such as microperimetry, a pre- and postoperative examination of the whole macular area in patients with ERM formation seems mandatory. Interestingly, macular thickness analysis alone showed no significant influence on postoperative VA improvement.

A limitation of our study is related to the limited number of patients included. In addition, OCT measurements were performed using horizontal scans only. One might hypothesize that additional vertical scans might provide more detailed information on IS/OS abnormalities and recovery.
In conclusion, we report that disruption of the photoreceptor IS/OS layer, especially in the parafoveal area evaluated by SD-OCT, is a predictor of VA in patients with ERM formation and provides additional information about the pathogenesis of VA decrease and functional surgical outcome in these patients. The presence of a normal IS/OS line indicates a morphological recovery of the photoreceptor layer following epimacular membrane surgery which is predictive for functional improvement. Morphological and functional tests should not be limited to the fovea, but should also include the parafoveal region.

\section{References}

1 Gandorfer A, Haritoglou C, Scheler R, Schumann R, Zhao F, Kampik A: Residual cellular proliferation on the internal limiting membrane in macular pucker surgery. Retina 2012;32:477-485.

- 2 Niwa T, Terasaki H, Kondo M, Piao CH, Suzuki T, Miyake Y: Function and morphology of macula before and after removal of idiopathic epiretinal membrane. Invest Ophthalmol Vis Sci 2003;44:1652-1656.

3 McCarty DJ, Mukesh BN, Chikani V, Wang JJ, Mitchell P, Taylor HR, McCarty CA: Prevalence and associations of epiretinal membranes in the visual impairment project. Am J Ophthalmol 2005;140:288-294.

-4 Michalewski J, Michalewska Z, Cisiecki S, Nawrocki J: Morphologically functional correlations of macular pathology connected with epiretinal membrane formation in spectral optical coherence tomography (SOCT). Graefes Arch Clin Exp Ophthalmol 2007;245: 1623-1631.

-5 Haritoglou C, Schumann RG, Kampik A, Gandorfer A: Heavy brilliant blue $\mathrm{G}$ for internal limiting membrane staining. Retina 2011; 31:405-407.

6 Inoue M, Morita S, Watanabe Y, Kaneko T, Yamane S, Kobayashi S, Arakawa A, Kadonosono K: Inner segment/outer segment junction assessed by spectral-domain optical coherence tomography in patients with idiopathic epiretinal membrane. Am J Ophthalmol 2010;150:834-839.

-7 Oster SF, Mojana F, Brar M, Yuson RM, Cheng L, Freeman WR: Disruption of the photoreceptor inner segment/outer segment layer on spectral domain-optical coherence tomography is a predictor of poor visual acuity in patients with epiretinal membranes. Retina 2010;30:713-718.
8 Spaide RF, Curcio CA: Anatomical correlates to the bands seen in the outer retina by optical coherence tomography: literature review and model. Retina 2011;31:1609-1619.

-9 Watanabe A, Arimoto S, Nishi O: Correlation between metamorphopsia and epiretinal membrane optical coherence tomography findings. Ophthalmology 2009;116:17881793.

10 Falkner-Radler CI, Glittenberg C, Hagen S, Benesch T, Binder S: Spectral-domain optical coherence tomography for monitoring epiretinal membrane surgery. Ophthalmology 2010;117:798-805.

11 Shimozono M, Oishi A, Hata M, Matsuki T, Ito S, Ishida K, Kurimoto Y: The significance of cone outer segment tips as a prognostic factor in epiretinal membrane surgery. Am J Ophthalmol 2012;153:698-704, 704.e1.

12 Itoh Y, Inoue M, Rii T, Hiraoka T, Hirakata A: Significant correlation between visual acuity and recovery of foveal cone microstructures after macular hole surgery. Am J Ophthalmol 2012;153:111-119.e1.

13 Inoue M, Arakawa A, Yamane S, Watanabe Y, Kadonosono K: Long-term outcome of macular microstructure assessed by optical coherence tomography in eyes with spontaneous resolution of macular hole. Am J Ophthalmol 2012;153:687-691.

14 Theodossiadis PG, Theodossiadis GP, Charonis A, Emfietzoglou I, Grigoropoulos VG, Liarakos VS: The photoreceptor layer as a prognostic factor for visual acuity in the secondary epiretinal membrane after retinal detachment surgery: imaging analysis by spectral-domain optical coherence tomography. Am J Ophthalmol 2011;151:973-980.

15 Shimozono M, Oishi A, Hata M, Kurimoto Y: Restoration of the photoreceptor outer segment and visual outcomes after macular hole closure: spectral-domain optical coherence tomography analysis. Graefes Arch Clin Exp Ophthalmol 2011;249:1469-1476.
16 Inoue M, Morita S, Watanabe Y, Kaneko T, Yamane S, Kobayashi S, Arakawa A, Kadonosono K: Preoperative inner segment/outer segment junction in spectral-domain optical coherence tomography as a prognostic factor in epiretinal membrane surgery. Retina 2011; 31:1366-1372.

17 Mitamura Y, Hirano K, Baba T, Yamamoto S: Correlation of visual recovery with presence of photoreceptor inner/outer segment junction in optical coherence images after epiretinal membrane surgery. $\mathrm{Br} \mathrm{J}$ Ophthalmol 2009;93:171-175.

-18 Sandali O, Basli E, Borderie V, Laroche L, Monin C: Recurrence of an idiopathic vasocentric epiretinal membrane: Clinical and surgical particularities. J Fr Ophtalmol 2012;35:48. e1-e5.

19 Yagi T, Sakata K, Funatsu H, Noma H, Yamamoto K, Hori S: Macular microcirculation in patients with epiretinal membrane before and after surgery. Graefes Arch Clin Exp Ophthalmol 2012;250:931-934.

20 Zhou J, Liu W, Li Q, Li X, Hao J, Wang XL: A comparative study of macular thickness and function before and after idiopathic macular epiretinal membrane surgery. Zhonghua Yan Ke Za Zhi 2012;48:119-123.

21 Kampik A: Clinical and pathological aspects of epiretinal membrane formation. Klin Monbl Augenheilkd 1982;181:386-387.

22 Kampik A: Pathology of epiretinal membrane, idiopathic macular hole, and vitreomacular traction syndrome. Retina 2012; 32(suppl 2):S194-S198.

23 Pesin SR, Olk RJ, Grand MG, Boniuk I, Arribas NP, Thomas MA, Williams DF, Burgess D: Vitrectomy for premacular fibroplasia. Prognostic factors, long-term follow-up, and time course of visual improvement. Ophthalmology 1991;98:1109-1114. 
24 Shiono A, Kogo J, Klose G, Takeda H, Ueno $\mathrm{H}$, Tokuda N, Inoue J, Matsuzawa A, Kayama N, Ueno S, Takagi H: Photoreceptor outer segment length: a prognostic factor for idiopathic epiretinal membrane surgery. Ophthalmology 2013, E-pub ahead of print.

25 Suh MH, Seo JM, Park KH, Yu HG: Associations between macular findings by optical coherence tomography and visual outcomes after epiretinal membrane removal. Am J Ophthalmol 2009;147:473-480 e473.

26 Rohrschneider K, Bultmann S, Springer C: Use of fundus perimetry (microperimetry) to quantify macular sensitivity. Prog Retin Eye Res 2008;27:536-548.
27 Karacorlu M, Ozdemir H, Senturk F, Karacorlu SA, Uysal O: Correlation of retinal sensitivity with visual acuity and macular thickness in eyes with idiopathic epimacular membrane. Int Ophthalmol 2010;30:285-290.

28 Arichika S, Hangai M, Yoshimura N: Correlation between thickening of the inner and outer retina and visual acuity in patients with epiretinal membrane. Retina 2010;30:503508.

29 Odrobina D, Michalewska Z, Michalewski J, Dziegielewski K, Nawrocki J: Long-term evaluation of vitreomacular traction disorder in spectral-domain optical coherence tomography. Retina 2011;31:324-331.

\$30 Haritoglou C, Neubauer AS, Reiniger IW, Priglinger SG, Gass CA, Kampik A: Longterm functional outcome of macular hole surgery correlated to optical coherence tomography measurements. Clin Experiment Ophthalmol 2007;35:208-213.
31 Wakabayashi T, Fujiwara M, Sakaguchi H, Kusaka S, Oshima Y: Foveal microstructure and visual acuity in surgically closed macular holes: spectral-domain optical coherence tomographic analysis. Ophthalmology 2010; 117:1815-1824.

32 Koo HC, Rhim WI, Lee EK: Morphologic and functional association of retinal layers beneath the epiretinal membrane with spectraldomain optical coherence tomography in eyes without photoreceptor abnormality. Graefes Arch Clin Exp Ophthalmol 2012;250: 491-498. 